\title{
Reactivity effect breakdown calculations with deterministic and stochastic perturbations analysis - JEFF-3.1.1 to JEFF3.2T1 (BRC-2009) actinides application
}

\author{
Y. Peneliau ${ }^{1, \mathrm{a}}$ and B. Morillon ${ }^{2}$ \\ 1 Commissariat à l'Énergie Atomique et aux Énergies Alternatives - Centre de Cadarache - 13108 \\ Saint-Paul-lez-Durance Cedex - France \\ 2 Commissariat à l'Énergie Atomique et aux Énergies Alternatives - Centre de Bruyères-le-Châtel - \\ 91297 Arpajon Cedex-France
}

\begin{abstract}
JEFF-3.1.1 is the reference nuclear data library in CEA for the design calculations of the next nuclear power plants. The validation of the new neutronics code systems is based on this library and changes in nuclear data should be looked at closely. Some new actinides evaluation files at high energies have been proposed by CEA/Bruyères-le-Chatel in 2009 and have been integrated in JEFF3.2T1 test release. For the new release JEFF-3.2, CEA will build new evaluation files for the actinides, which should be a combination of the new evaluated data coming from BRC-2009 in the high energy range and improvements or new evaluations in the resolved and unresolved resonance range from CEA-Cadarache. To prepare the building of these new files, benchmarking the BRC-2009 library in comparison with the JEFF-3.1.1 library was very important. The crucial points to evaluate were the improvements in the continuum range and the discrepancies in the resonance range. The present work presents for a selected set of benchmarks the discrepancies in the effective multiplication factor obtained while using the JEFF-3.1.1 or JEFF-3.2T1 library with the deterministic code package ERANOS/PARIS and the stochastic code TRIPOLI4. They have both been used to calculate cross section perturbations or other nuclear data perturbations when possible. This has permittted to identify the origin of the discrepancies in reactivity calculations. In addition, this work also shows the importance of cross section processing validation. Actually, some fast neutron spectrum calculations have led to opposite tendancies between the deterministic code package and the stochastic code. Some particular nuclear data (MT $=5$ in ENDF terminology) seem to be incompatible with the current MERGE or GECCO processing codes.
\end{abstract}

\section{Introduction}

Different units are involved at CEA in the production of nuclear data for the neutronics simulation codes. Each one is specialized in a particular energy range and all contribute to improve the JEFF nuclear data through their participation to the JEFF project. It is today asked to these units to collaborate and to produce a unique evaluation file for CEA.

The actual reference nuclear data library is JEFF-3.1.1 [1] and the validation work is based on this library for the next GEN-IV nuclear power plants and for the sodium fast reactor prototype ASTRID (for Advanced Sodium Technological Reactor for Industrial Demonstration). The changes in the nucear data may have a huge impact on this work and they have to be looked at very closely. So, as a first step in the collaborative work, the impact of the nuclear data change from JEFF-3.1.1 to BRC-2009 [2] for some U, Pu and Am actinides only (proposed in JEFF-3.2T1 library released in 2011) is invertigated.

\footnotetext{
a e-mail: yannick.peneliau@cea.fr
} 
To have a detailed information on the origin of the discrepancies, some reactivity effects breakdown are performed for some benchmarks of interest with the deterministic code package PARIS [3] and with the stochastic code TRIPOLI-4 [4]. The nuclear data processing is performed by CEA for the different codes, for both JEFF-3.1.1 and JEFF3.2T1 libraries.

This article first presents the selected set of benchmarks. Then, it details the different methods used in the reactivity effect breakdown analysis. In a third part, it shows some results in reactivity dicrepancies to illustrate the main findings of this work and the choices to which they have led in the new evaluation files, which will be proposed in JEFF-3.2 library.

\section{Selected set of benchmarks}

In order to cover the whole energy range and all actinides, some thermal, intermediate and fast spectrum benchmarks for Uranium, Plutonium or Mixed fuels are selected. The benchmarks are either taken from the ICSBEP [5] criticality experiments database or from the IRPhE [6] reactor experiments database.

The ICSBEP benchmarks are taken from the HMF, IMF and HST series for uranium and from PMF and PST series for plutonium. Additional HxI intermediate spectrum benchmarks have also been calculated in order to test ${ }^{235} U$ and ${ }^{238} U$ in softer spectra (closer to SFR ones). The reactor benchmarks are the MAS1B uranium configuration in the french reactor MASURCA and the 7A and 7B plutonium configurations in the german reactor SNEAK.

\section{Fine analysis methods}

\subsection{Deterministic method}

The deterministic method used in the analysis of the nuclear data is based on the exact perturbation theory. Actually, the reactivity breakdown decomposition can be assessed per isotop, per nuclear reaction type and per energy group.

Considering the direct flux $\Phi$ and the adjoint flux $\Phi^{+}$, the transport, removal and diffusion operator $\mathrm{A}$ and the fission operator $\mathrm{F}$, the transport equation is :

$$
\left(A-\frac{F}{k_{\text {eff }}}\right) \Phi=0
$$

with :

$A \Phi(\mathbf{r}, \mathbf{\Omega}, E)=\mathbf{\Omega} \cdot \nabla \Phi(\mathbf{r}, \mathbf{\Omega}, E)+\Sigma_{t} \Phi(\mathbf{r}, \boldsymbol{\Omega}, E)-\iint \Sigma_{s}\left(\mathbf{r}, E^{\prime} \rightarrow E, \mathbf{\Omega}^{\prime} \rightarrow \mathbf{\Omega}\right) \Phi\left(\mathbf{r}, \mathbf{\Omega}^{\prime}, E^{\prime}\right) \times d \mathbf{\Omega}^{\prime} \times d E^{\prime}$

and :

$$
F \phi(\mathbf{r}, \mathbf{\Omega}, E)=\frac{\chi(E)}{4 \pi} \times \iint v_{f}\left(\mathbf{r}, E^{\prime}, \mathbf{\Omega}^{\prime} \Phi\left(\mathbf{r}, \mathbf{\Omega}^{\prime}, E^{\prime}\right) \times d \mathbf{\Omega}^{\prime} \times d E^{\prime}\right.
$$

Then, the reactivity variation $d \rho$ can be written :

$$
d \rho=\frac{d k_{e f f}}{k_{e f f}^{2}}=-\frac{<\Phi^{+},\left(d A-\frac{d F}{k_{e} f f}\right) \Phi>}{<\Phi^{+}, F \Phi>}
$$

where the scalar product $<., .>$ has the classical definition.

Then, the reactivity change from state (1) to state (2) is obtained by the formula :

$$
\delta \rho_{(1) \rightarrow(2)}=-\frac{\left.<\Phi_{(1)}^{+},\left(\delta A-\frac{\delta F}{k_{e} f f}\right) \Phi_{(2)}>\right)}{<\Phi_{(1)}^{+}, F \Phi_{(2)}>}
$$

The PARIS package performs this calculations. The ECCO cell code is dedicated to the calculation of the homogenized and collapsed multigroup cross sections and the SNATCH Sn core calculation code performs the direct and adjoint angular fluxes $\phi(\mathbf{r}, \boldsymbol{\Omega}, E)$ and $\phi^{+}(\mathbf{r}, \boldsymbol{\Omega}, E)$. 


\subsection{Monte Carlo methods}

This study used three Monte Carlo methods to analyze the discrepancies between one evaluation (BRC-2009) and the other one (JEFF-3.1.1).

The first method is very simple. It consists simply in modifying the TRIPOLI-4 isotops' general dictionary by adding one or some fictive new isotop(s) (with new nuclear data) in order to replaced the original one(s).

The second method is quite particular to TRIPOLI-4. Actually, the simulation code needs, as nuclear data, the evaluation ASCII file and a pointwise cross sections ASCII or BINARY file. So, it is possible to replace a whole ENDF format block by another one $(\mathrm{MF}=1, \mathrm{MT}=456$ for the number of prompt fission neutrons for example) to perform a new simulation. This may typically be applied to independent data like number of fission neutrons, fission spectra, some anisotropy data $((v, \chi$, pdfs $f(\mu)$ ).

The third method is the correlated samples Monte Carlo techniques. It consists in simulating the particles with a set of nuclear data, and to consider this simulation as a biased simulation for another set of nuclear data (called perturbed set). Each time the data of the perturbed set is different than the one of the original set and is involved in the probability density function used in a sampled event, a "perturbed set" correction weight, defined as the ratio of the probability density functions, is calculated and taken into account in the estimation of perturbed results.

\section{Reactivity effects analysis}

This section is dedicated to the highlighting of the results of the BRC-2009 library, compared to the JEFF-3.1.1 library. It also focuses on the reactivity breakdown analysis, with the PARIS deterministic package and the TRIPOLI-4 Monte Carlo code.

\subsection{ICSBEP Uranium benchmarks}

Concerning the uranium fuel, it is very important to ensure that the actual validation of the CEA neutronics codes and their nuclear data libraries is not affected by the BRC-2009 changes. In this part, the fast and intermediate spectra are tested. Figure 1 shows graphically the effect of BRC-2009 library for the HMF and IMF series. The standard deviation for JEFF-3.1.1 or BRC-2009 calculations is close to $10 \mathrm{pcm}$. These results shows that the JEFF-3.1.1 tends to underestimate the experimental values, and that BRC-2009 corrects this underestimation (the mean value of the correction is $+280 \mathrm{pcm}$, and the value goes from +150 to $+360 \mathrm{pcm}$ ). Concerning intermediate enrichment, the Big Ten benchmark (IEU-MET-FAST 007) is also better estimated whereas the JEMINA (IEU-MET-FAST 001) change is not significant, compared to the experimental value. The mean effect is $+480 \mathrm{pcm}$, from +240 to +780 ).

To illustrate the fine analysis methods used in the study, Table 1 shows the reactivity (or $\mathrm{k}_{e f f}$ in the case of TRIPOLI-4) effect of the library change per isotop and reaction type for the HEU-MET-FAST 001 benchmark. It should be noted that $(n, X n)$ is only $(n, 2 n)$ in TRIPOLI-4 calculation and that the total effects (in bold font) are obtained with two independent simulations. For TRIPOLI-4 results, the standard deviation in pcm is given between brackets. A zero value means that it was less than 0,5.

For the $(n, n)$ reaction (elastic diffusion), the anisotropy is taken into account in the TRIPOLI-4 calculation. The global result is the combination of a punctaul elastic cross section perturbation with an elastic anisotropy modification ( $\mathrm{MF}=4, \mathrm{MT}=2$ replacement in evaluation file) leading to -382 (9) pcm.

The global effect of the use of the BRC-2009 evaluation files is a mean increase of 280 to $300 \mathrm{pcm}$ for fast and intermediate spectra benchmarks and a few dozens pcm for thermal spectrum. This has led to propose a new ${ }^{235} U$ evaluation with a continuum energy range taken from BRC-2009 and thermal and resonance energy ranges taken from the existing JEFF-3.1.1.

No change has been stated for the ${ }^{238} U$ evaluation yet from this study. 


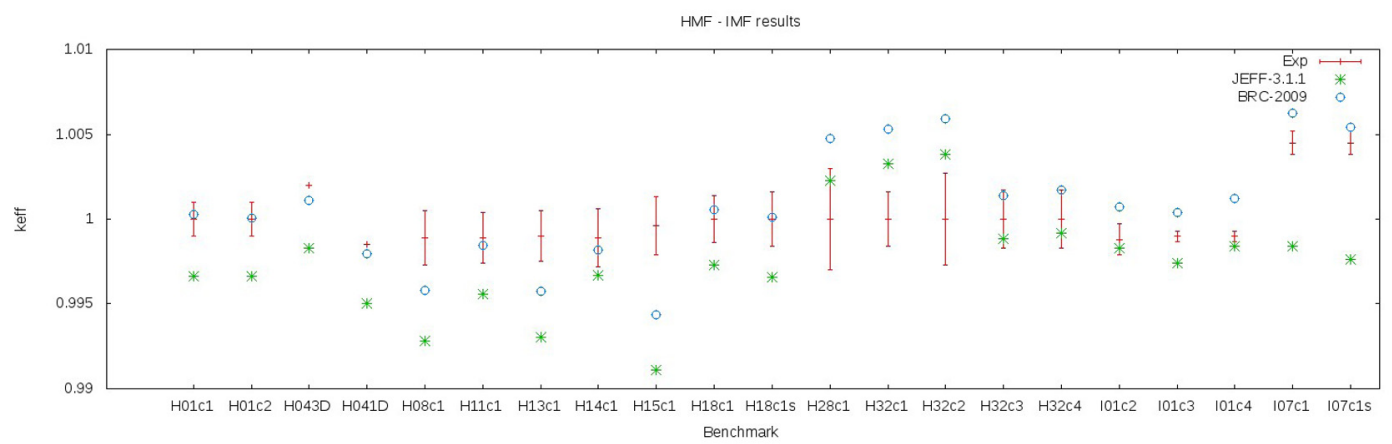

Fig. 1. HMF and IMF TRIPOLI-4 results

Table 1. HMF001 benchmark reactivity breakdown analysis

\begin{tabular}{lllllllll}
\hline Isotope & $(\mathrm{n}, \gamma)$ & $v$ & $\chi$ & $(\mathrm{n}, \mathrm{f})$ & $(\mathrm{n}, \mathrm{n})$ & $\left(\mathrm{n}, \mathrm{n}^{\prime}\right)$ & $(\mathrm{n}, \mathrm{Xn})$ & Total \\
\hline \hline${ }^{235} \mathrm{U}$ & & & & & & & \\
\hline PARIS & 127 & -838 & 2 & 323 & 8 & 558 & 192 & $\mathbf{+ 3 7 1}$ \\
TRIPOLI-4 & $130(1)$ & $-835(13)$ & $\mathrm{x}$ & $310(2)$ & $26(9)$ & $\mathrm{x}$ & $156(2)$ & $\mathbf{+ 3 6 5}(\mathbf{1 4})$ \\
Discrepancy & $+3(1)$ & $+3(13)$ & $\mathrm{x}$ & $-13(2)$ & $+18(9)$ & $\mathrm{x}$ & $36(2)$ & $\mathbf{- 6}(\mathbf{1 4})$ \\
\hline${ }^{238} \mathrm{U}$ & & & & & & & & \\
\hline PARIS & 4 & -28 & 1 & 22 & -15 & 7 & 7 & $\mathbf{- 2}$ \\
TRIPOLI-4 & $4(0)$ & $-16(13)$ & $\mathrm{x}$ & $24(1)$ & $-17(2)$ & $\mathrm{x}$ & $0(0)$ & $\mathbf{+ 1 4 ( 1 4 )}$ \\
Discrepancy & $0(0)$ & $+12(13)$ & $\mathrm{x}$ & $+2(1)$ & $-2(2)$ & $\mathrm{x}$ & $-7(0)$ & $\mathbf{+ 1 6 ( 1 4 )}$ \\
\hline Total & & & & & & & & \\
\hline PARIS & 131 & -866 & 3 & 345 & -7 & 565 & 199 & $\mathbf{+ 3 6 9}$ \\
TRIPOLI-4 & $\mathrm{x}$ & $\mathrm{x}$ & $\mathrm{x}$ & $\mathrm{x}$ & $\mathrm{x}$ & $\mathrm{x}$ & $\mathrm{x}$ & $\mathbf{+ 3 7 9}(\mathbf{2 0})$ \\
Discrepancy & $\mathrm{x}$ & $\mathrm{x}$ & $\mathrm{x}$ & $\mathrm{x}$ & $\mathrm{x}$ & $\mathrm{x}$ & $\mathrm{x}$ & $\mathbf{+ 1 0}(\mathbf{2 0})$ \\
\hline
\end{tabular}

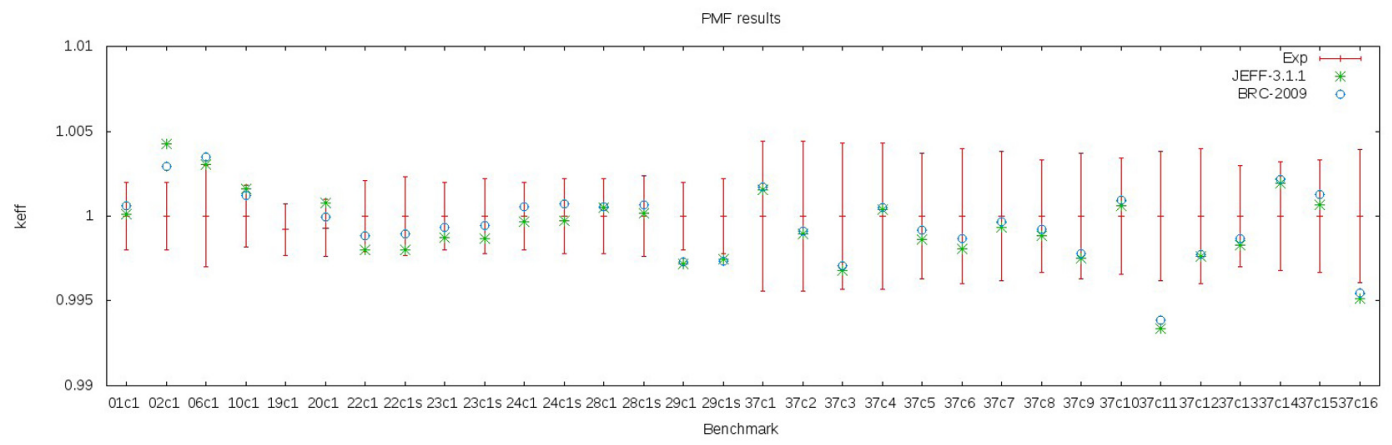

Fig. 2. PMF TRIPOLI-4 results

\subsection{ICSBEP Plutonium benchmarks}

The results for the plutonium and fast spectrum benchmarks taken from the ICSBEP database are shown in figure 2.

An identical fine analysis has been performed for the PMF001 benchmark. The results are shown in table 2.

The final effects are very small for the fast spectrum. The mean discrepancy is about $+30 \mathrm{pcm}$, and the variation goes from $-130 \mathrm{pcm}$ to $+100 \mathrm{pcm}$. The PMF002 benchmark (highly enriched with ${ }^{240} \mathrm{Pu}$ ) 
Table 2. PMF001 benchmark reactivity breakdown analysis

\begin{tabular}{lllllllll}
\hline Isotope & $(\mathrm{n}, \gamma)$ & $v$ & $\chi$ & $(\mathrm{n}, \mathrm{f})$ & $(\mathrm{n}, \mathrm{n})$ & $(\mathrm{n}, \mathrm{n})$ & $(\mathrm{n}, \mathrm{Xn})$ & Total \\
\hline \hline${ }^{239} \mathrm{Pu}$ & & & & & & & & \\
\hline PARIS & -80 & 89 & 5 & 20 & -93 & 160 & 28 & $\mathbf{+ 1 2 9}$ \\
TRIPOLI-4 & $-78(0)$ & $\mathrm{x}(\mathrm{x})$ & $\mathrm{x}$ & $4(1)$ & $-92(8)$ & $\mathrm{x}$ & $10(1)$ & +() \\
Discrepancy & $+2(0)$ & $\mathrm{xx}(\mathrm{xx})$ & $\mathrm{x}$ & $-16(1)$ & $+1(8)$ & $\mathrm{x}$ & $-18(1)$ & +() \\
\hline${ }^{240} \mathrm{Pu}$ & & & & & & & & \\
\hline PARIS & -14 & -70 & -2 & 32 & -7 & 8 & 1 & $\mathbf{- 2}$ \\
TRIPOLI-4 & $-14(0)$ & $\mathrm{xxx}(\mathrm{xx})$ & $\mathrm{x}$ & $35(1)$ & $-7(1)$ & $\mathrm{x}$ & $0(0)$ & +() \\
Discrepancy & $0(0)$ & $\mathrm{xxx}(\mathrm{xx})$ & $\mathrm{x}$ & $+3(1)$ & $0(1)$ & $\mathrm{x}$ & $-1(0)$ & +() \\
\hline Total & & & & & & & & \\
\hline PARIS & -94 & 19 & 3 & 52 & -100 & 168 & 29 & $\mathbf{+ 7 7}$ \\
TRIPOLI-4 & $\mathrm{x}$ & $\mathrm{x}$ & $\mathrm{x}$ & $\mathrm{x}$ & $\mathrm{x}$ & $\mathrm{x}$ & $\mathrm{x}$ & $\mathbf{+ 5 3 ( 1 1 )}$ \\
Discrepancy & $\mathrm{x}$ & $\mathrm{x}$ & $\mathrm{x}$ & $\mathrm{x}$ & $\mathrm{x}$ & $\mathrm{x}$ & $\mathrm{x}$ & $\mathbf{- 2 4}(\mathbf{1 1})$ \\
\hline
\end{tabular}

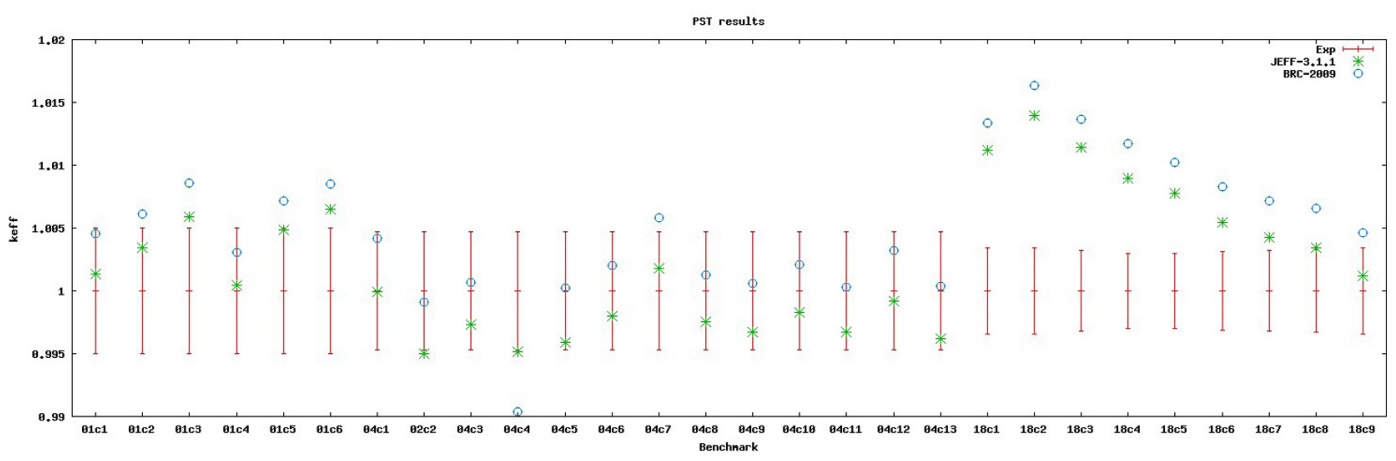

Fig. 3. PST TRIPOLI-4 results

shows a -130 pcm decrease, closer to the experimental value. For thermal spectrum benchmarks, the effects are more important, about $+300 \mathrm{pcm}$. The results are shown in figure 3 .

A new ${ }^{239} \mathrm{Pu}$ evaluation based on data form BRC-2009 for the continuum energy range and from JEFF-3.1.1 and recent ORNL work [7] for the thermal and resonance energy range will be proposed for the new JEFF-3.2 library. Concerning ${ }^{240} \mathrm{Pu}$, the BRC-2009 evaluation will be the CEA reference evaluation, despite fast spectrum ${ }^{240} \mathrm{Pu}$ highly enriched (close to PWR recycling enrichment, i.e. $25 \%$ ) benchmarks should be considered.

\subsection{Reactor benchmarks}

A few experimental reactor configurations have been tested in order to evaluate the new BRC-2009 evaluation files in softer spectra than the ICSBEP fast spectra. The results are shown in table 3, the values between brackets are the TRIPOLI- 4 standard deviations expressed in $\mathrm{pcm}$.

The results show inconsistencies between TRIPOLI-4 and ERANOS-2 calculated effects. An academic benchmark based on the HEU-MET-FAST 001 configuration with high ${ }^{238} U$ content and same density has been used to investigate the origin of this inconsistency. Table 4 shows the results. A fine study in which the reactivity effect breakdown was used has shown that the MT=5 cross section has produced an error in the ECCO cell code. The dedicated MERGE or GECCO processing tools are involved. 
Table 3. Experimental reactors TRIPOLI-4 results

\begin{tabular}{llll}
\hline Experiment & JEFF-3.1.1 & BRC-2009 & Discrepancy $(\mathrm{pcm})$ \\
\hline \hline PARIS & & & \\
\hline MAS-1B & 0,99965 & 1,00465 & +490 \\
SNEAK-7A & 1,00625 & 1,00769 & +144 \\
SNEAK-7B & 1,00311 & 1,00328 & +17 \\
\hline TRIPOLI-4 & & & \\
\hline MAS-1B & $1,00447(21)$ & $1,00979(22)$ & $+532(30)$ \\
SNEAK-7A & $1,00913(9)$ & $1,01093(9)$ & $+180(13)$ \\
SNEAK-7B & $1,00398(8)$ & $1,00665(8)$ & $+267(11)$ \\
\hline
\end{tabular}

Table 4. Academic benchmark : JEFF-3.1.1 to JEFF-3.2T $1{ }^{238} U$ effect results

\begin{tabular}{llll}
\hline Code & $5 \%{ }^{238} U$ & $50 \%{ }^{238} U$ & $89 \%{ }^{238} U$ \\
\hline \hline Critical rdius (cm) & 8,7406 & 12,605 & 34,165 \\
TRIPOLI-4 & $+339(14)$ & $+328(18)$ & $+691(17)$ \\
PARIS & +363 & $+291(40)$ & +282 \\
\hline
\end{tabular}

\section{Outlook and conclusion}

The study presented here has shown the capability of TRIPOLI-4 to be an efficient tool in perturbation analysis for evaluation files comparison. Its capabilities should be extended with an automatic procedure to build the perturbation data from two pointwise cross sections files, with probability tables data.

The work has also shown the necessity to validate the ECCO processing in the case of PARIS, and more generally the processing of the cell code library.

The validation (and the reactivity effect breakdown in particular) procedures presented here will be implemented in a tool dedicated to evaluators. The aim of this tool will be the automatic calculation of the impact of new changes in an evaluation file on a user selected set of benchmarks, user selected reference library, for all codes (deterministic or stochastic ones). The nuclear data processing and the calculations will be automatically performed by this tool.

\section{References}

1. A. Santamarina et al., The JEFF-3.1.1 Nuclear Data Library - JEFF Report 22 - Validation results from JEF-2.2 to JEFF-3.1.1 (OECD/NEA, 2009)

2. P. Romain, New Neutron evaluations of actinides with the TALYS code : first validation tests JEFDOC 1266, (2008)

3. R. Letellier, C. Suteau, D. Fournier, J.M. Ruggieri, High Oreder Ordinate Transport in Hexagonal Geometry : a New Capability in ERANOS Il Nuovo Cimento, 33C, (2010)

4. E. Brun, E. Dimonteil, F.X. Hugot, N.Huot, Y.K. Lee, F. Malvagi, A. Mazzolo, O. Petit, J.C. Trama, A. Zoia Overview of TRIPOLI-4 version 7 Continuous-energy Monte Carlo Transport Code Proceedings of ICAPP 2011, Nice, France, (2011)

5. OECD/NEA, ICSBEP : International Criticality and Safety Benchmark Evaluation Project September 2005 Edition NEA/NSC/DOC(93)03, (2005)

6. OECD/NEA, International Handbook of Evaluated Reactor Physics Benchmark Experiments March 2010 Edition NEA/NSC/DOC(2006)1, (2010)

7. L. Leal, Pu-239 Resonance Evaluation JEF-DOC 1432, (2012) 